\title{
Parametric Nonlinear Model Reduction for Structural Dynamics
}

\author{
Christopher Lerch* Christian H. Meyer** \\ * Technical University of Munich, Chair of Automatic Control, \\ Boltzmannstr. 15, 85748 Garching b. München, Germany (e-mail: \\ christopher.lerch@tum.de). \\ ** Technical University of Munich, Chair Applied Mechanics, \\ Boltzmannstr. 15, 85748 Garching b. München, Germany (e-mail: \\ christian.meyer@tum.de).
}

Keywords: Nonlinear model reduction; Subspace methods; Real-time control;

\section{INTRODUCTION}

The finite element method has grown to the standard way to solve partial differential equations numerically. In the area of structural dynamics this method discretizes the partial differential equation

$$
\nabla \cdot \boldsymbol{\sigma}+\hat{\boldsymbol{b}}-\varrho \ddot{\boldsymbol{u}}=\mathbf{0} \text { on } \Omega
$$

with boundary and initial conditions $\boldsymbol{u}=\hat{\boldsymbol{u}}$ on $\partial \Omega_{u}$, $\boldsymbol{\sigma} \cdot \boldsymbol{n}=\hat{\boldsymbol{t}}$ on $\partial \Omega_{\sigma}, \boldsymbol{u}\left(t_{0}\right)=\boldsymbol{u}_{0}$ and $\dot{\boldsymbol{u}}\left(t_{0}\right)=\dot{\boldsymbol{u}}_{0} \cdot \boldsymbol{u}$ are the displacements of the structure. If the analyzed structure undergoes large deflections, the outcoming discretized equation of motion

$$
\boldsymbol{M} \ddot{\boldsymbol{u}}(t)+\boldsymbol{f}(\boldsymbol{u}(t))=\boldsymbol{B} \boldsymbol{F}(t)
$$

is nonlinear with respect to the restoring force $\boldsymbol{f}(\boldsymbol{u})$.

In applications such as design or control, the equation of motion can depend on parameters that concern the shape, material and boundary conditions as illustrated in Fig. 1. These parameters can either be changed by the engineer during construction (usually shape and material) or due to operation conditions and control (usually boundary conditions).

The dependence of Eq. (1) on these parameters can be expressed by

$$
\boldsymbol{M}(\boldsymbol{p}) \ddot{\boldsymbol{u}}(t)+\boldsymbol{f}(\boldsymbol{p}, \boldsymbol{u}(t))=\boldsymbol{B}(\boldsymbol{p}) \boldsymbol{F}(t)
$$

where $\boldsymbol{p} \in \mathbb{P}$ is a set of variable parameters.

Finite element models of complex geometries that appear in engineering can have million degrees of freedoms which

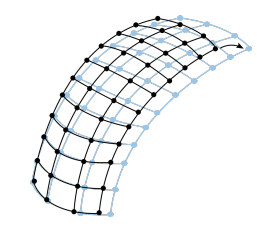

Shape

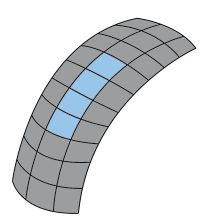

Material

Parameter Set $p$

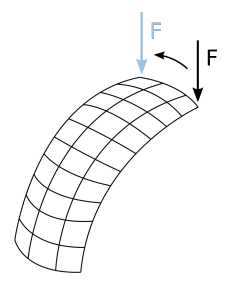

Boundary Cond.
Fig. 1. Parameterization of Structural Finite Element Models.

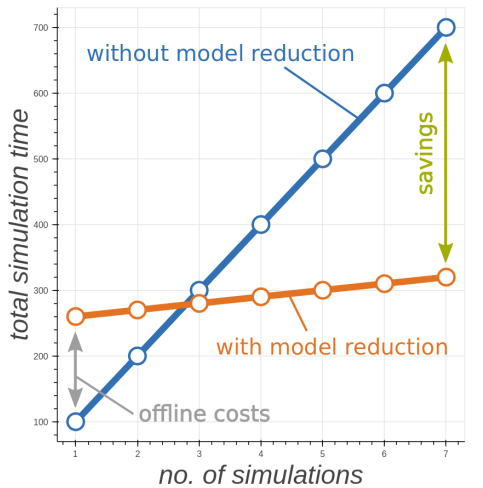

Fig. 2. Computational savings with model reduction.

can lead to high computation costs when solving Eq. (1). In applications such as design iterations or realtime control where the equation of motion have to be solved several times, a short simulation time is highly demanded. One approach to satisfy this demand is model reduction. It reduces the computation time by reducing the problem dimension in a reduction step (offline) and then computing the reduced smaller model (online). After a certain number of simulations or calculated timesteps the total simulation time can be reduced as illustrated in Fig. 2.

\section{MODEL REDUCTION FOR FINITE ELEMENT MODELS IN STRUCTURAL DYNAMICS}

For equations of type (1) the model reduction is performed in two steps. First, the number of unknowns is reduced by a Galerkin projection. Second, the evaluation of the nonlinear force term is sped up by hyperreduction.

\subsection{Galerkin Projection}

In order to reduce the dimension of the problem, i.e. the number of unknowns, a Galerkin projection is performed. Therein a lower-dimensional approximation of the relevant system dynamics is done by

$$
\boldsymbol{u}=\boldsymbol{V} \boldsymbol{q}+\varepsilon \approx \boldsymbol{V} \boldsymbol{q}
$$

with the nonlinear reduction basis $\boldsymbol{V}=\left[\boldsymbol{V}_{\text {lin }} \mid \boldsymbol{V}_{\text {nl }}\right]$ consisting of a linear part $\boldsymbol{V}_{\text {lin }}$ augmented with an additional nonlinear part $\boldsymbol{V}_{\mathrm{nl}}$. The linear part is calculated 
Table 1. Possible combinations of methods for calculation of the nonlinear reduction basis.

\begin{tabular}{lll}
\hline & Linear part $\boldsymbol{V}_{\text {lin }}$ & Nonlinear part $\boldsymbol{V}_{\mathrm{nl}}$ \\
\hline Modal truncation & Vibration modes & $\begin{array}{l}\text { Modal derivatives } \\
\text { Static derivatives }\end{array}$ \\
Moment matching & Krylov directions & $\begin{array}{l}\text { Krylov derivatives } \\
\text { Static derivatives }\end{array}$ \\
Other linear methods & Linear basis vectors & $\begin{array}{l}\text { Exact derivatives } \\
\text { Static derivatives }\end{array}$ \\
\hline
\end{tabular}

using linear methods together with a linearized model (e.g. Salimbahrami and Lohmann (2006)). The additional nonlinear part consists of exact or static derivatives of the linear basis vectors accounting for the nonlinear behavior (e.g. Idelsohn and Cardona (1984)). Tab. 1 lists basic combinations of methods that are used.

This leads to the projected and reduced system dynamics

$$
\boldsymbol{V}^{\top} \boldsymbol{M} \boldsymbol{V} \ddot{\boldsymbol{q}}(t)+\boldsymbol{V}^{\top} \boldsymbol{f}(\boldsymbol{V} \boldsymbol{q}(t))=\boldsymbol{V}^{\top} \boldsymbol{B} \boldsymbol{F}(t) .
$$

The quality of the reduction basis and the associated reduced system are evaluated and optimized via a specific $\mathcal{H}_{2}$-norm for the error system.

\subsection{Hyperreduction}

Although the projection reduces the dimension of the problem, its reduction of computation time is quite poor. This originates from the evaluation of the reduced nonlinear restoring force $\boldsymbol{V}^{\top} \boldsymbol{f}(\boldsymbol{V} \boldsymbol{q})$, because it still has to be evaluated in the full element domain, i.e. for the physical displacements $\boldsymbol{u}=\boldsymbol{V} \boldsymbol{q}$. Hyperreduction methods have been developed to overcome this issue.

The ansatz of Hyperreduction is to evaluate the elemental restoring forces $\boldsymbol{f}_{e}$ of a subset of all elements and extrapolate their contribution to the global restoring force vector $f$ instead of assembling the contibution of all elements:

$$
\begin{aligned}
\boldsymbol{V}^{\top} \boldsymbol{f}(\boldsymbol{V} \boldsymbol{q}) & =\sum_{e \in E} \boldsymbol{V}^{\top} \boldsymbol{B}_{e}^{\top} \boldsymbol{f}_{e}\left(\boldsymbol{B}_{e} \boldsymbol{V}_{e} \boldsymbol{q}\right) \\
& \approx \sum_{e \in \widetilde{E} \subset E} \boldsymbol{V}^{\top} \boldsymbol{L}_{e} \boldsymbol{B}_{e}^{\top} \boldsymbol{f}_{e}\left(\boldsymbol{B}_{e} \boldsymbol{V}_{e} \boldsymbol{q}\right) .
\end{aligned}
$$

Different Hyperreduction methods differ in the way the element subset $\widetilde{E}$ and the operator $\boldsymbol{L}_{e}$ are chosen. One of the most popular Hyperreduction methods for structural problems is called the Energy Conserving Sampling and Weighting (ECSW) method. It has advantageous properties when applied to mechanical problems, such as stability and passivity (Farhat et al. $(2014,2015)$ ).

\section{PARAMETRIC REDUCTION}

The consideration of parameter changes only needs a prior suitable sampling of the parameter space $\mathbb{P}$. Then the same methods can be applied for each parameter sampling point $\boldsymbol{p}_{i} \in \mathbb{P}(i=1,2, \ldots, N)$ resulting in $N$ different reduction bases $\boldsymbol{V}\left(\boldsymbol{p}_{i}\right)$ and if necessary reduced systems $\mathcal{S}_{\mathrm{r}}\left(\boldsymbol{p}_{i}\right)$ (e.g. Benner et al. (2015)). Tab. 2 summarizes basic approaches used within this framework.
Table 2. Methods used for parametric reduction.

\begin{tabular}{l|l}
\hline Local approaches & Global approaches \\
\hline Basis updating $\boldsymbol{V}\left(\boldsymbol{p}_{i}\right)$ & Concatenation to global basis \\
& {$\left[\begin{array}{l}\boldsymbol{V}\left(\boldsymbol{p}_{1}\right) \boldsymbol{V}\left(\boldsymbol{p}_{2}\right) \cdots \boldsymbol{V}\left(\boldsymbol{p}_{N}\right) \\
\text { Basis interpolation } \boldsymbol{V}\left(\boldsymbol{p}_{i}\right) \\
\text { Global parameter-dependent } \\
\text { basis } \boldsymbol{V}(\boldsymbol{p})\end{array}\right.$} \\
Matrix/system interpolation $\mathcal{S}_{\mathrm{r}}\left(\boldsymbol{p}_{i}\right)$ & \\
\hline
\end{tabular}

\section{RESEARCH CODE AMFE}

Nonlinear model reduction methods have not been implemented in commercial code so far. Furthermore, the implementation of new developed methods is very tedious in conjunction with commercial finite element code. Therefore, an open source research code, called AMfe (Rutzmoser (2017)), has been implemented that makes prototyping new model reduction methods easy. The code is written in Python and provides easy access to internal computations due to its modular structure. Several reduction methods have been implemented such as methods for calculating reduction bases and hyperreduction methods e.g. ECSW.

\section{OUTLOOK}

At current state only non-parametric nonlinear model order reduction methods have been implemented in the research code. The goal of current research of the authors is to develop and implement new reduction techniques for parameter dependent systems for applications such as design, optimization and control systems.

\section{ACKNOWLEDGEMENTS}

The authors thank the German Research Foundation (DFG) for supporting this research project within the priority programm (SPP) 1897 "Calm, Smooth and Smart: Novel Approaches for Influencing Vibrations by Means of Deliberately Introduced Dissipation".

\section{REFERENCES}

Benner, P., Gugercin, S., and Willcox, K. (2015). A survey of projection-based model reduction methods for parametric dynamical systems. SIAM Review, 57(4), 483-531.

Farhat, C., Avery, P., Chapman, T., and Cortial, J. (2014). Dimensional reduction of nonlinear finite element dynamic models with finite rotations and energy-based mesh sampling and weighting for computational efficiency. Int. J. for Numerical Methods in Engineering, 98(9), 625-662.

Farhat, C., Chapman, T., and Avery, P. (2015). Structure-preserving, stability, and accuracy properties of the energy-conserving sampling and weighting method for the hyper reduction of nonlinear finite element dynamic models. International Journal for Numerical Methods in Engineering, 102(5), 1077-1110.

Idelsohn, S. and Cardona, A. (1984). Recent advances in reduction methods in non linear structural dynamics. In Proceedings of the 2nd International Conference on Recent Advances in Structural Dynamics 1984, 475-482. University of Southampton, England.

Rutzmoser, J. (2017). Amfe - finite elements with simplicity in mind. https://github.com/AppliedMechanics/AMfe.

Salimbahrami, S. and Lohmann, B. (2006). Order reduction of large scale second-order systems using Krylov subspace methods. Linear Algebra and its Applications, 415(2), 385-405. 